\title{
Home Tele-monitoring: Economic and Clinical Impact of the Service for Patients with Chronic Respiratory Disease
}

\author{
Christian Rainero ${ }^{1}$, Silvana Secinaro ${ }^{1}$, Elena Nave $^{2}$ \& Elisabetta Bignamini ${ }^{2}$ \\ ${ }^{1}$ Department of Management, University of Turin, Turin, Italy \\ ${ }^{2}$ S.C. Pediatric Pulmonology, Regina Margherita Children Hospital, AOU Città della Salute e della Scienza, Turin, \\ Italy \\ Correspondence: Christian Rainero, Associate Professor, Department of Management, University of Turin, Italy.
}

Received: October 19, 2017

Accepted: November 14, 2017

Online Published: November 21, 2017

doi:10.5430/jms.v8n5p48

URL: https://doi.org/10.5430/jms.v8n5p48

\begin{abstract}
The term "telemedicine" defines "the use of electronic communications technology to provide and support health care for infants, children, adolescents, and young adults when distance separates the practitioner from the patient, parent, guardian, or referring practitioner" (AAP 2004, 639).

The pilot study has been involved 71 patients with a telemedicine service of the Unit of respiratory insufficiency of the Regina Margherita Children's Hospital of Turin follows actually and their caregiver. The scopes are mainly two: the evaluation the potential of control system of functional examination in the various expression of disease, and improvement the knowledge of the perceived quality of the service according the service users, in according to the economic and ethical impact. These variables are able to determine the actual usefulness of the home recorder-ICT device prescription at discharge of these types of patients, or when occur the conditions of necessity, or in case of Centre take charge of new patients. Patients assessed effectiveness home tele-monitoring service and patient's satisfaction through semi-structured questionnaires.

The administering of the questionnaires took place during the day hospital, or during office visits/outpatient visits, or during occasional hospitalizations.

The analysis is extended to the economic and budgetary aspects, focusing on comparisons of spending between traditional medicine and telemedicine, investigating whether there is a correlation between patient satisfaction and reduced spending by telemedicine

The analysis of the data showed interesting results in order to improve the type of service offered to these patients, also in term of economic and social results.
\end{abstract}

Keywords: telemedicine, respiratory disease, healthcare management, costs and benefits

\section{Introduction}

The research aims to highlight the clinical, economic and ethical impact in the assistance of telemedicine services, by focus on home care for cystic fibrosis patients and technology dependent children.

Telemedicine technology embodies the electronic acquisition processing, dissemination, storage, retrieval, and exchange of information for the purpose of promoting health, preventing disease, treating the sick, managing chronic illness, rehabilitating the disabled, and protecting public health and safety (Bashshur \& Shannon 2009).

Within the umbrella expression "e-health", meaning use of Internet to provide health care (Eng 2001), the term "telemedicine" defines, in the pediatrics context, "the use of electronic communications technology to provide and support health care for infants, children, adolescents, and young adults when distance separates the practitioner from the patient, parent, guardian, or referring practitioner" (AAP 2004, 639).

The term "telemonitoring" is refers to "home programmed control" of the parameters of some vital functions. It concerns the measurement, the record and the secure transmission of medical data through a suitable home digital recording and transmitting device. Physicians of the specialized Centre can thus see patient data daily, with a direct communication, and intervene in case of clinical complication. Such service it is not use for the home exacerbations (but possibly as an aid to exacerbations diagnosis) and does not provide for emergency/urgency interventions. 
The benefits to be derived for health care professionals - for example accessing and sharing information and experiences - for telemedicine, e- health device and service as a field of successful investment, for reducing health costs, are all acquisitions that will affect improvement therapeutic offer aimed at chronic patients.

This pilot study focuses on e-health ICT innovation, specifically in telemedicine form, like effective tool to increase patients and parents' empowerment. This initiative falls within the wider context of integrative patient centred care, in which the patient and his/her caregivers are regarded as self-determining active agents.

\section{Background}

\subsection{Economic Background}

Telemonitoring has gained attention thanks to its potential of reducing morbidity and mortality, healthcare utilisation and costs. However, its benefit with regard to clinical and economic outcomes remains to be clearly demonstrated. A pilot study in German demonstrated that telemonitoring is a viable strategy to reduce mortality, healthcare costs and utilisation at 12 months. Contrary to widespread fear, reducing the intensity of care does not seem to impact unfavourably on health outcomes. The evidence offers strong support for introducing telemonitoring as a component of case management (Achelrod D et al, 2016).

We reviewed previous studies available in literature on the issue of economic viability of home telemonitoring. One of this (Seto, 2008) analysed the existing economic evidence to determine whether home telemonitoring of patients with congestive heart failure results in decreased costs. She analysed data from a total of ten different home telemonitoring studies. Nine of these studies analysed the direct costs to the healthcare system. All of them found cost reductions from telemonitoring compared with usual care, ranging from 1.6 percent to 68.3 percent. The cost reductions were mainly associated with savings from reduced expenditures on hospitalizations and, to a lesser extent, home visits.

A second study (Polinesa et al, 2009) also reviewed the literature on the cost-effectiveness of home telehealth for chronic disease. Their review included economic assessments such as cost minimization analyses, cost-effectiveness analyses, cost utility analyses and cost benefit analyses. A total of twenty-two empirical studies were found to be relevant for inclusion in the database, most of which were from the United States and a majority focused on patients with congestive heart failure. Half of the included studies $(n=11)$ were concerned specifically with home telemonitoring interventions (as defined above) while the other half referred to other forms of home telehealth programs such as telephone case management and video-based nursing consultations. As in the previous review, usual care varied across the studies and the home telehealth interventions tended to be complex, often with differences in more than one facet of patient management in the intervention phase of the study. Most economic studies involved an assessment of the costs associated with specific healthcare resources, including hospitalizations and emergency department visits. The authors found that, in most studies (ten of eleven), home telemonitoring led reduced spending on healthcare resources.

A third study (Paré et al, 2013), indicated significant benefits to the home telemonitoring program as evidenced by large reductions in number of hospitalizations, length of average hospital stay, and, to a lesser extent, number of emergency room visits. Contrary to expectations, however, the number of home visits by nurses increased both during and after the telemonitoring intervention. In terms of the financial analysis, the tele-homecare program resulted in significant savings. Importantly, they observed that these effects were more significant for heart failure and COPD (Chronic Obstructive Pulmonary Disease patients). They concluded that while the present economic analysis led to positive results, additional assessments should be conducted to confirm the cost-effectiveness of this mode of care delivery.

\subsection{Clinical Background}

Chronic respiratory patients requiring oxygen or home mechanical ventilation experience frequent exacerbations and hospitalizations with related costs. Strict monitoring and care have been recommended.

(Vitacca et al 2009).

In children, the most frequent causes of respiratory failure are neurological diseases (particularly neuromuscular diseases), metabolic and genetic syndrome and cystic fibrosis.

Cystic fibrosis (CF) is the most common lethal autosomal recessive genetic disease in Caucasians, but it should be noted that in multi-racial Europe, no ethnic group is exempt from the disease, although prevalence varies across the continent and has been extensively studied for many decades. 
During the past two decades, care for European patients with CF has been increasingly organized in specialized centres and diagnoses generally reported to regional or national registries. This facilitates determination of the prevalence of CF for most European countries The prognosis of cystic fibrosis has improved substantially so that now more than half of the patient population is in the adult age range in most of the European Countries. Estimates of the extent of this improvement should allow health care facilities to be planned to deal with the expanding adult population of patients with cystic fibrosis. Clinically characterized by progressive lung disease, nearly $90 \%$ of patients with $\mathrm{CF}$ have exocrine pancreatic insufficiency and subsequent malabsorption that lead to a risk for malnutrition secondary to increased losses from malabsorption and increased energy demands from infections, chronic disease, and decreased oral intake. End-stage lung disease is the principal cause of death.

Compliance is a major problem in CF. It is estimated that compliance of nebulized medications varies between 60-30\%. Adolescents and young adults are particularly prone to reduced compliance as they transition from childhood where responsibility for all aspects of their care was their parents. The burden of medical interventions in $\mathrm{CF}$ can be significant and the young adult can find it difficult to integrate their regime of medications and physiotherapy into their daily routine. ICT could be a useful tool in the armamentarium of the physician to improve compliance and to feed information back to the clinicians about respiratory status (pulse oximetry, spirometry) and allows interception of respiratory exacerbation.

In chronic respiratory failure other than cystic fibrosis, children on oxygen or home mechanical ventilation, can present benefits from telemedicine program regarding prevention of hospitalizations, improving of quality of life reduction of unscheduled/emergency center visits, and minor hospital length of stay with an hypothetic rationalization of healthcare costs.

\section{The Pilot Study}

The Unit of respiratory insufficiency of the Regina Margherita Children's Hospital of Turin follows 71 patients with a telemedicine service, specifically with a service of health status/data home monitoring and tracking. These patients are individuals with chronic disease that need regular monitoring because the nature of their health condition.

The pilot study has been involved these patients and their caregiver, and the scopes are mainly two: the evaluation the potential of control system of functional examination in the various expression of disease, and improvement the knowledge of the perceived quality of the service according the service users. These variables can determine the actual usefulness of the home recorder-ICT device prescription at discharge of these types of patients, or when occur the conditions of necessity, or in case of Centre take charge of new patients.

The involved subjects are mainly patients and pediatrics patients with cystic fibrosis or, generally, with chronic respiratory insufficiency and complex disease with invasive or not invasive ventilation (mostly neuromuscular disease).

The first class includes competent people who are able to decide for themselves. The second includes individuals judged constitutively and objective unable to make their own decisions, the minors. The involvement of these individual implies the involvement of their legal representatives and their caregivers. We already know that the role of the caregiver is in most cases covered by the women; in this specific context by the mothers.

Patients involved in the service are also suffering from various diseases besides cystic fibrosis, and feature frameworks by highly variable clinical severity. They belong to the large set of encephalopathies or neuromuscular disorder. (Note 1) The wide variability of the pathologies of which the patients are suffering is added to those related to the age range, to the disease phase (especially for cystic fibrosis) and to the geographical location of patients' home.

Patients, and their caregiver when the age was less than eighteen years, assessed effectiveness home telemonitoring service and patient's/caregiver's satisfaction through semi-structured questionnaires. They, in according with the Medical Home Policy Statement (2002), are made to understand and empathize with the feelings and perspectives of the family as well as the child or youth.

The analysis of the data showed interesting results in order to improve the type of service offered to these patients.

\section{Method and Data}

In July 2015, the recruited patients have been all the 71 patients and pediatric patients follow with the home-telemonitoring service from the Regional Centre of Turin and from the Unit of respiratory insufficiency (Regina Margherita Children's Hospital). Two patients died before the end of this pilot study. 
The compiled questionaries' have been 43, of which 9 of patients and caregivers that have interrupted data transmission, abandoning thus the telemedicine project because don't fulfilled or because followed from another Centre, or whose broadcasts were discontinued, and 34 about patients and caregivers still participants to the project.

The only inclusion criterion has been: "patients assisted with home telemonitoring service, also in the case in which the service has been interrupted or discontinued". The only exclusion criterions have been: "patients (or caregivers for pediatric patients) which have refusal to participate in the study" and "unavailable home phone land line". The kind of the sample is summarized in Figure 1.

The location data

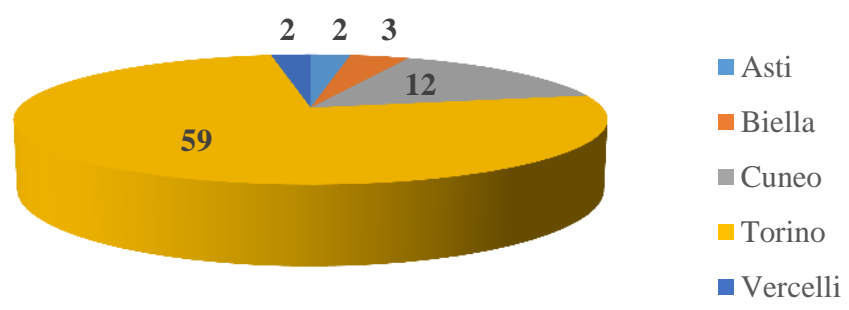

Figure 1

Own source

The administering of the questionnaires took place during the day hospital, or during office visits/outpatient visits, or during occasional hospitalizations. In some cases, questionnaires were sent by mail. Before being enrolled in the project, each adult patient signed a consent form; in case of minors, the legal representative signed it. Interrogate patients, and in the case of minors over the ages of 13 years also their caregivers, allowed to see some telemedicine service aspects otherwise unobservable.

\section{Ethical Perspective}

In this home-telemonitoring health service, patients' bodily integrity will not be violated at any stage. It does not provide for the collection of biological samples of any kind, nor the use of invasive procedures, but rather the production, collection and processing of data on patients' body features. The intent is to maximize the benefits and minimize the risks of medical treatment favoring the ability for patients to remain at home as the disease conditions permit it. The physical interventions involved in this research are the spirometry and the oxygen saturation They do not violate the patient's body integrity because they do not provide any form of bodily invasion. They do not cause any form of physical pain or suffering, and discomfort experienced possibly can refer to the psychological feeling of control that the sending of measurements of physiological states in the Centre may result. This risk is covered stressing repeatedly to patients their freedom to withdraw their participation at any time without having to suffer any consequences

Among the needs that associations of patients have indicated, some express specifically moral needs. Is called "moral need" the kind of need that is intimately bound up with personal conceptions of good and right. It is arising from the moral perspective that the individual chooses to direct his/her lives and choices. All choices of patients and caregivers about care, and care management, presuppose a moral view, personal beliefs, value conceptions. In health care and research, moral autonomy of patients and volunteers is protected, and it is expressed in their freedom of self-determination. Patients' and volunteers' moral autonomy is protected just a particular instance of individual moral autonomy. Need analysis thus involves analyzing which of them express peculiarly a moral need - according to some "an upper-level need, the highest level" (Maslow 1943). 
The set "moral need" include the need to share own moral responsibility with others, the need to exercise fully self-determination freedom, and the need of being in relationship. A service that innovative solution is able to offer, is also determined by the answers that the services is able to give to moral needs of patients and caregiver.

The analysis also included the views expressed by pediatric patients from the age of twelve. They have too developed will, preferences and needs that are able to reveal if given them the opportunity to express themselves.

\section{Results and Discussion}

\subsection{Clinical Results}

The objectives, which are valid from an ethical point of view, that the introduction of ICT devices for the care of chronically ill patients should center, are mainly three: promoting patient, pediatric patient (and caregiver when the pediatric patient is very young or her/his condition is very severe) empowerment through the self-management of his/her conditions, the increase of the quality of life and the reduction of the treatment costs, i.e. the saving in the allocation of a scarce resource.

The encephalopathies or neuromuscular disorder, as well as cystic fibrosis, are disease that lead to a significant deterioration of quality of life both in patients (Péntek et al 2014; Fidika et al 2015, Smith Grzvwacz 2014). How parents cope with the burden of caring for a chronically ill child depends on various factors: contextual psychological, cultural, emotional, social. Contextual factors include the possible ways and times to cure the disease. Home cure monitoring via ICT devices can act like protective effects, can increase support provided to parents, improving the quality of their life and increasing parental perceived control or their parental competence.

The introduction of ICT devices in the health care raises furthermore, according to bioethics literature or European Guide Line, two main ethical concerns: the possible deterioration of the doctor-patient relationship is the first (Commission to the European Parliament on Telemedicine, 2008) and the possible creation of "a 2-tiered system in which patients who are able to pay are granted in-person access and poor children are treated by telemedicine" (AAP 2004) is the second.

The telemonitoring experience in this Italian healthcare setting does not raise such concerns. Patients and caregivers have not perceived any loss of quality in their patient-doctor relationships, and patients and caregivers does not fear that this could happen in the future. The second concern, however, is currently not relevant in the framework of this health care setting because all health services directed to these groups of patients are held free of charge.

Regard those who have abandoned the use of telemonitoring, they have done it for three orders of reasons: technical reasons (because followed by another center or because of malfunction problems encountered) personal reasons (home telemonitoring is considered useless or bored the patient) and moral reasons (violates the freedom or it is a burden to the routine or increases the concern).

The results of data survey analysis showed the need for patients and caregivers to be able to see themselves their sending data. Now the procedure does not allow such acquisition: only the doctors to which arrived the shipped data can see the actual patients' values. In terms of self-determination, empowerment of patient and skills development, this need seems justified and should receive an appropriate response.

The major discomforts detected are due to technical reasons. The ICT devices wheezing production, its mechanics voice, the wires that in the night twist patients and disturb his/her sleep are some of the problematic aspects of the fruition of this service. This means that in patients' and caregivers' perception the most problems related to home telemonitoring are technical, not ethical. Improve the functionality of the ICT device implies improving the appreciation from the users of the service, and then the regularity of their use.

Questioning parents and children was very interesting because often the answers to the same questions differ. The two vision often do not coincide. What is considered a problem for someone, it is not for another. In regard to the involvement of pediatric patients in care choices, this disparity in their visions spurs to require a more direct participation of minors to allow to their voice to be most listened on the various aspects concerning their health and their body.

\subsection{Economical Results}

The economic data analysis was developed with the comparison of the two-year costs of activities of the pulmonology department, considering hospitalization data, patient data, direct operating costs, adjusted by indirect operating costs, which were not considered for the analysis of the case. Management costs, be they direct or indirect, were divided between traditional activities and activities in telemedicine. The results (Table 1) show the direct costs per patient day at the traditional support of around 1.200,00 euro, compared to the daily cost of 10 telemedicine 
patient euro. The clear difference in costs, to the benefit of telemedicine is however filled by the frequency of hospitalizations by patients in telemedical assistance: the average days of telemedicine patients hospitalization is $20 \%$ higher than the patients in the traditional care.

Table 1

\begin{tabular}{|c|c|c|c|}
\hline & Data in Euro & 2014 & 2015 \\
\hline Hospital direct costs (excluding tele-monitoring patients) per year & & $3.142 .945,42$ & $2.991 .036,74$ \\
\hline Hospital direct costs for patients per day & & $1.194,1282$ & $1.200,25551$ \\
\hline Direct telemonitoring costs per patients per day & & 9,78 & 5,83 \\
\hline Hospitalization costs for telemonitoring patients per year & & $33.435,59$ & $22.804,85$ \\
\hline
\end{tabular}

Own source

So, at the cost level of analysis, the use of telemedicine, in the two years sample, resulting in neutral. The dividing line is the ethical and clinical appearance. The analysis deserves further study in a longer span of time, to test the effects of the introduction of telemedicine on reducing the use of shelters.

\section{Conclusion and Advances}

The study shows that the evaluation of the service for young adults and pediatric patients with chronic respiratory disease by telemonitoring covers three aspects: clinical, ethical and economic. The evaluation the potential of control system of functional examination in the various expression of disease, and improvement the knowledge of the perceived quality of the service according the service users, in according to the economic and ethical impact. These variables are able to determine the actual usefulness of the home recorder-ICT device prescription at discharge of these types of patients, or when occur the conditions of necessity, or in case of Centre take charge of new patients. Patients assessed effectiveness home tele-monitoring service and patient's satisfaction through semi-structured questionnaires. On a scale of influence, the study shows that the determining factors are the clinical and ethical aspect, revealing a neutral from an economic point of view. The economic aspect is influenced positively if it is a net reduction in hospital admissions in terms of number of patients, but especially in terms of hospital days. The results of data survey analysis showed the need for patients and caregivers to be able to see themselves their sending data. This means that in patients' and caregivers' perception the most problems related to home telemonitoring are technical, not ethical.

At the cost level of analysis, the use of telemedicine, in the two years sample, resulting in neutral. The dividing line is the ethical and clinical appearance. The analysis deserves further study in a longer span of time, to test the effects of the introduction of telemedicine on reducing the use of shelters.

More research is needed to define the ethical and clinical aspects of telemedicine that probably overcome the economic logic. The prospect of development is to evaluate the effects of telemedicine under the three profiles, in a broader 5-7 years time frame.

\section{Acknowledgements}

The Authors thank Loredana Cannata for her previous work on this topic.

\section{References}

Achelrod, D., et al. (2016, Oct.). Health-economic evaluation of home telemonitoring for COPD in Germany: evidence from a large population-based cohort. European Journal of Health Economics, 3.

American Academy of Pediatrics. (2002). The Medical Home - Policy Statement. Pediatrics, 110(1), 184-186. https://doi.org/10.1542/peds.110.1.184

American Academy of Pediatrics. (2004). Telemedicine: Pediatric Application - Technical Report. Pediatrics, 113(6), 639-643. https://doi.org/10.1542/peds.113.6.e639

Bashshur, R.L., \& Shannon, G.W. (2009). National Telemedicine Initiatives: Essential to Healthcare Reform. Telemedicine and e-Health. https://doi.org/10.1089/tmj.2009.9960 
Communication Commission to the European Parliament on Telemedicine for the benefit of patients, healthcare systems and society, 4. 11. 2008.

Eng, T.R. (2001). The eHealth Landscape: a Terrain Map of Emerging Information and Communication Technologies in Health and Health Care. The Robert Wood Johnson Foundation, Princeton.

Fidika, A., Herle, M., Lehmann, C., Weiss, C., Knaevelsrud, C., \& Goldbeck, L. (2015). A web-based psychological support program for caregivers of children with cystic fibrosis: a pilot study. Health Qual Life Outcomes, 13(11). https://doi.org/10.1186/s12955-015-0211-y

Guy Paré, Placide Poba-Nzaou, \& Claude Sicotte. (2013, March). Home telemonitoring for chronic disease management: An economic assessment. International Journal of Technology Assessment in Health Care. https://doi.org/10.1017/S0266462313000111

Maslow, A.H. (1943). A theory of human motivation. Psychological Review, 50, 370-396. https://doi.org/10.1037/h0054346

Péntek, M., Kosztolányi, G., Melegh, B., Halász, A., Pogány, G., Baji, P., ... Gulácsi, L. (2014). Health related quality of life and disease burden of patients with cystic fibrosis and their caregivers: Results of the European BURQOL-RD survey in Hungary. Orv Hetil, 155(42), 1673-84. https://doi.org/10.1556/OH.2014.30016

Polinesa, J., Coyle, D., Coyle, K., \& McGill, S. (2009). Home telehealth for chronic disease management: Systematic review and an analysis of economic evaluations. Int J Technol Assess Health Care, 25, 339-349. https://doi.org/10.1017/S0266462309990201

Royal College of Phisicians. (2000). Guidelines for the Ethical Conduct of Medical Research Involving Children. Archives of Disease in Childhood, 82(2), 177-182. https://doi.org/10.1136/adc.82.2.177

Seto, E. (2008). Cost comparison between telemonitoring and usual care of heart failure: A systematic review. Telemed J E Health, 14, 679-686. https://doi.org/10.1089/tmj.2007.0114

Smith, A.M., \& Grzvwacz, J.G. (2014). Health and well-being in midlife parents of children with special health needs. Fam Syyst Health, 32(3), 303-12. https://doi.org/10.1037/fsh0000049

Vitacca, M., Bianchi, L., Guerra, A., Fracchia, C., Spanevello, A., Balbi, B., \& Scalvini, S. (2009). Tele-assistance in chronic respiratory failure patients: a randomised clinical trial. Eur Respir J., 33, 411. https://doi.org/10.1183/09031936.00005608

\section{Note}

Note 1. They include: encephalopathy, congenital myopathy, SMARD, SMA2, DMD, syndrome Charge, Myastenia gravis, interstitial lung disease, Steinert dystrophy. 